\title{
HILBERT TRANSFORMS, FRACTIONAL INTEGRATION AND DIFFERENTIATION
}

BY P. L. BUTZER AND W. TREBELS

Communicated by R. C. Buck, August 10, 1967

The purpose of this note is to announce a number of results concerning the various approaches to fractional integration on the real line $E$ as due to H. Weyl [9], M. Riesz [7], W. Feller [4] and G. O. Okikiolu [6]. Our principal contributions are on extensions of theorems of J. L. B. Cooper [3], on the interchange of the operations of fractional integration (differentiation) and the Hilbert transform, on the counterpart of a theorem of $\mathrm{H}$. Weyl [9] on periodic functions to the real line, and on partial differential equations of fractional order.

Since Fourier transform methods are mainly used in the proofs, the discussion is restricted to the space $L^{p}(E)$ for $1 \leqq p \leqq 2$. Full details of the work reported here as well as further results will appear in a monograph to be published by the Westdeutscher Verlag, Opladen.

Let $f \in L^{p}(E), 1 \leqq p \leqq 2$. We define the Hilbert transform of $f$ by

$$
H_{0} f(x)=\mathrm{PV} \frac{1}{\pi} \int_{-\infty}^{\infty} \frac{f(t)}{x-t} d t,
$$

Weyl's fractional integral of order $1-\alpha$ and the analogous integral of M. Riesz [7] by

$$
\begin{aligned}
f_{1-\alpha}(x) & =\frac{1}{\Gamma(1-\alpha)} \int_{-\infty}^{x} \frac{f(t)}{(x-t)^{\alpha}} d t, \\
I_{1-\alpha} f(x) & =C_{0}(\alpha) \int_{-\infty}^{\infty} \frac{f(t)}{|x-t|^{\alpha}} d t,
\end{aligned}
$$

respectively, where $C_{0}^{-1}(\alpha)=2 \Gamma(1-\alpha) \cos (\pi / 2)(1-\alpha)$. We slightly modify M. Riesz' integral for functions $\mu$ of normalized bounded variation on $E$, i.e. $\mu \in \mathrm{BV}(E)$ :

$$
I_{1-\alpha} \mu(x)=C_{0}(\alpha) \int_{-\infty}^{\infty}\left(1 /|x-t|^{\alpha}\right) d \mu(t) .
$$

Following G. O. Okikiolu [6] we consider further the integral

$$
\tilde{I_{1-\alpha}} f(x)=C_{s}(\alpha) \int_{-\infty}^{\infty} \frac{\operatorname{sgn}(x-t)}{|x-t|^{\alpha}} f(t) d t,
$$


where $C_{s}^{-1}(\alpha)=2 \Gamma(1-\alpha) \sin (\pi / 2)(1-\alpha)$ and analogously $\tilde{I_{1-\alpha} \mu}$ in case $\mu \in \mathrm{BV}(E)$. The integrals (2), (3), (5) exist a.e. if $1-1 / p<\alpha<1$, while the Stieltjes integrals exist a.e. for $0<\alpha<1$.

A differentiation of fractional order $\alpha$ of an $L^{p}$-function may be defined either by the derivative of $(2)$, thus

$$
f^{(\alpha)}(x)=\left(d^{[\alpha]+1} / d x^{[\alpha]+1}\right) f_{1-\{\alpha\rangle}(x)
$$

whenever the right side exists or by

$$
f^{\{\alpha\}}(x)=\left(d^{[\alpha]+1} / d x^{[\alpha]+1}\right) I_{1-\{\alpha\}} f(x) \quad(1-(1 / p)<\langle\alpha\rangle<1),
$$

where $\alpha>0,[\alpha]$ being the largest integer $\leqq \alpha$ and $\langle\alpha\rangle=\alpha-[\alpha]$. Denoting the Fourier transform of $f \in L^{p}(E)$ and $\mu \in \operatorname{BV}(E)$ by $f^{\wedge}(v)$ and $\mu^{\nu}(v)$, respectively, it will be useful to define the following function classes:

$$
\begin{aligned}
V_{\alpha}^{p}=\left\{f \in L^{p}(E) ; \quad(i \operatorname{sgn} v)(i v)^{[\alpha]} \mid\right. & \left.v\right|^{\langle\alpha\rangle f^{\wedge}(v)} \\
& =\left\{\begin{array}{l}
\mu^{\sim}(v), \mu \in \mathrm{BV}(E), p=1 \\
g_{\wedge}(v), g \in L^{p}(E), 1<p \leqq 2
\end{array}\right\}
\end{aligned}
$$

and

$$
\begin{aligned}
V_{\alpha}^{\sim^{p}}=\left\{f \in L^{p}(E) ;(i v)^{[\alpha]}|v|^{\langle\alpha\rangle f^{\wedge}(v)}\right. & \\
& =\left\{\begin{array}{l}
\mu^{\nu}(v), \mu \in \mathrm{BV}(E), p=1 \\
g^{\wedge}(v), g \in L^{p}(E), 1<p \leqq 2
\end{array}\right\} .
\end{aligned}
$$

The following theorem contains generalizations of results due to J. L. B. Cooper [3] and P. L. Butzer [2]. Here we set

$$
\Delta_{h}^{n} g(x)=\sum_{k=0}^{n}(-1)^{k}\left(\begin{array}{l}
n \\
k
\end{array}\right)(g(x+(n-k) h) .
$$

THEOREM 1. If $f \in L^{p}(E), 1 \leqq p \leqq 2,\langle\alpha\rangle \neq 0$, then the following assertions are equivalent:

(a) $f \in V_{\alpha}^{p}$;

(b) $f, f^{\prime}, \cdots, f^{([a]-1)}$ are locally absolutely continuous on $E$, $f^{([\alpha])} \in L^{p}(E)$ and

$$
\begin{aligned}
& \left\|C_{c}(\langle\alpha\rangle) \int_{-\infty}^{\infty}\left\{\frac{1}{|\cdot+h-t|\langle\alpha\rangle}-\frac{1}{|\cdot-t|\langle\alpha\rangle}\right\} f^{([\alpha])}(t) d t\right\|_{p} \\
& \equiv\left\|I_{1-\langle\alpha) f}^{h} f^{([\alpha])}\right\|_{p}=O(|h|) \quad(h \rightarrow 0) ;
\end{aligned}
$$


(c) $\left\|\Delta_{h}^{[\alpha]} I_{1-\langle\alpha) f}^{h}\right\|_{p}=O\left(\mid h^{[\alpha]+1}\right) \quad(h \rightarrow 0)$;

(d) $\| H_{0}\left(C_{s}(\langle\alpha\rangle) \int_{-\infty}^{\infty}\left\{\frac{\operatorname{sgn}(\cdot+h-t)}{|\cdot+h-t|\langle\alpha\rangle}-\frac{\operatorname{sgn}(\cdot-t)}{|\cdot-t|\langle\alpha\rangle}\right\} f^{([\alpha])(t) d t) \|_{p}}\right.$ $\equiv\left\|H_{0}\left(I_{1-\langle\alpha\rangle}^{\sim h}{ }^{([\alpha])}\right)\right\|_{p}=O(|h|) \quad(h \rightarrow 0) ;$

(e) $\| \Delta_{h}^{[\alpha]} H_{0}\left(I_{1-\langle\alpha\rangle f)}^{\sim h} \|_{p}=O\left(|h|^{[\alpha]+1}\right) \quad(h \rightarrow 0)\right.$.

The analog of this theorem also holds for the class $V_{\alpha}^{\sim p}$. The equivalence of (b) and (d) (or (c) and (e)) is based upon the fact that $I_{1-\beta}^{h} g(x)=H_{0}\left(I_{1-\beta}^{\sim h} g\right)(x)$ a.e., $0<\beta<1$, by which one may prove

Proposition 1. If $f \in L^{p}(E), p>1$ and $1-(1 / p)<\beta<1$, then

$$
\tilde{I_{1-\beta}} f(x)=H_{0}\left(I_{1-\beta} f\right)(x)=I_{1-\beta}\left(H_{0} f\right)(x) \quad \text { a.e. }
$$

The next two propositions are concerned with the differentiability of the integral (3) and the interchange of the operations of fractional differentiation and integration.

Proposition 2. If $f \in L^{p}(E), 1<p \leqq 2$ and $1-(1 / p)<\langle\alpha\rangle<1$, then $f^{\{\alpha\}} \in L^{p}(E)$ iff $\left(H_{0} f\right)^{\{\alpha\}} \in L^{p}(E)$. Furthermore,

$$
\begin{aligned}
H_{0} f^{\{\alpha\}}(x) & =H_{0}\left(\frac{d}{d u} I_{1-\langle\alpha\rangle} f^{([\alpha])}(u)\right)(x)=\frac{d}{d x} I_{1-\langle\alpha\rangle}\left(H_{0} f^{([\alpha])}\right)(x) \\
& =\frac{d}{d x} I_{1-\langle\alpha\rangle}\left(H_{0} f\right)^{([\alpha])}(x)=\left(H_{0} f\right)^{\{\alpha\}}(x) \quad \text { a.e. }
\end{aligned}
$$

Proposition 3. Let $f \in L^{p}(E), 1 \leqq p \leqq 2,1-(1 / p)<\langle\alpha\rangle<1$ and $f^{(\alpha)} \in L^{p}(E)$. Then

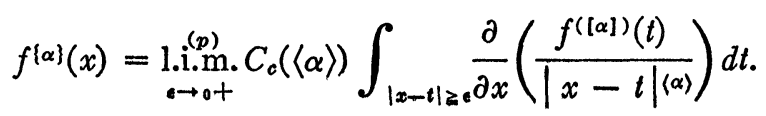

Applying a relation of $\mathrm{L}$. von Wolfersdorf [8], valid for $f \in L^{p}(E)$, $1<p \leqq 2,1-(1 / p)<\beta<1$, one may express the Weyl integral (2) as a linear combination of the Riesz integral of $f$ and $H_{0} f$, thus

$$
f_{1-\beta}(x)=\cos \frac{\pi}{2}(1-\beta) I_{1-\beta} f(x)+\sin \frac{\pi}{2}(1-\beta) I_{1-\beta}\left(H_{0} f\right)(x) \text { a.e., }
$$

and correspondingly for the Riesz integral (3),

$$
I_{1-\beta} f(x)=\cos \frac{\pi}{2}(1-\beta) f_{1-\beta}(x)-\sin \frac{\pi}{2}(1-\beta)\left(H_{0} f\right)_{1-\beta}(x) \quad \text { a.e., }
$$


equations which were also shown by $H$. Kober [5] using other methods. This gives

$$
\left(H_{0} f\right)_{1-\beta}(x)=H_{0} f_{1-\beta}(x) \text { a.e. }
$$

It now follows that the definitions (6) and (7) of fractional differentiation are equivalent. Indeed,

THEOREM 2. Let $f \in L^{p}(E), 1<p \leqq 2$ and $1-(1 / p)<\langle\alpha\rangle<1$. Then $f^{(\alpha)} \in L^{p}(E)$ iff $f^{\{\alpha\}} \in L^{p}(E)$, which in turn holds iff $f \in V_{\alpha}^{p}\left(\right.$ or $\left.V_{\alpha}^{\sim p}\right)$.

Proposition 4. Let $f \in L^{p}(E), 1<p \leqq 2$ and $1-1 / p<\langle\alpha\rangle<1$. Then $f^{(\alpha)} \in L^{p}(E)$ iff $\left(H_{0} f\right)^{(\alpha)} \in L^{p}(E)$. Furthermore $H_{0} f^{(\alpha)}(x)=\left(H_{0} f\right)^{(\alpha)}(x)$ a.e.

Of further interest are conditions an $L^{1}$-function $f$ must fulfill in order to admit a representation of the type $f(x)=I_{\alpha} \mu(x)$. We have

THEOREM 3. Let $f \in L^{1}(E)$. Then

$$
\left\|\int_{e}^{\infty} t^{-(1+\alpha)}\{f(\cdot+t)-2 f(\cdot)+f(\cdot-t)\} d t\right\|_{1}=O(1)
$$

uniformly in $\epsilon>0$

iff there exists a $\mu \in \mathrm{BV}(E)$ with $f(x)=I_{\alpha} \mu(x)$ a.e. for $0<\alpha<1$, or $\left(H_{0} f\right)(x)=\mu(x)$ a.e. for $\alpha=1$ and $(d / d x) f(x)=I_{\alpha-1}^{\sim} \mu(x)$ a.e. for $1<\alpha<2$. Furthermore, (8) holds iff $f \in V_{\alpha}^{\sim}$ for $0<\alpha<1, f \in V_{\alpha}^{p}$ for $1 \leqq \alpha<2$.

An analogous theorem holds for $1<p \leqq 2$. We conclude with the counterpart of a theorem of $\mathrm{H}$. Weyl [9].

THEOREM 4. If $f \in L^{1}(E)$ and $I_{\alpha} \mu(x)=f(x)$ a.e., then $\|f(\cdot+h)-f(\cdot)\|_{1}$ $=O\left(|h|^{\alpha}\right), 0<\alpha<1$, i.e. $f \in \operatorname{Lip}(\alpha, 1)$. Conversely, if $f \in \operatorname{Lip}(\alpha, 1)$, there exists a $\nu \in \mathrm{BV}(E)$ with $f(x)=I_{\beta} \nu(x)$ a.e., where $0<\beta<\alpha<1$. If $\|f(\cdot+h)-2 f(\cdot)+f(\cdot-h)\|_{1}=O(|h|)$, then $f(x)=I_{\beta} \nu(x)$ a.e., $0<\beta<1$.

With the help of these results we may solve the equation

$$
\frac{\partial}{\partial t} u(x, t)=-\left(\frac{\partial}{\partial x} H_{0}\right)^{k} I_{1-\beta} u(x, t), \quad \lim _{t \rightarrow 0+} u(x, t)=f(x),
$$

$k=1,2, \cdots, 0<\beta \leqq 1, I_{0}=$ identity operator (which for $k=2, \beta=1$ is the classical heat conduction equation) and obtain the generalized Weierstrass singular integral as the solution. This gives a further interpretation of the Bochner [1] operator 


$$
\begin{aligned}
-\left(-\frac{d^{2}}{d x^{2}}\right)^{\alpha / 2} & =-\left(\frac{d}{d x} H_{0}\right)^{k} I_{1-\beta} \\
(0 & <\alpha-k=\beta \leqq 1, \quad k=1,2, \cdots \cdot)
\end{aligned}
$$

Another interpretation is due to W. Feller [4].

\section{REFERENCES}

1. S. Bochner, Quasi-analytic functions, Laplace operator, positive kernels, Ann. of Math. (2) 51 (1950), 68-91.

2. P. L. Butzer, Beziehungen zwischen den Riemannschen, Taylorschen und gewöhnlichen Ableitungen reellwertiger Funktionen, Math. Ann. 144 (1961), 275-298.

3. J. L. B. Cooper, Some problems in the theory of Fourier transforms, Arch. Rational Mech. Anal. 14 (1963), 213-216.

4. W. Feller, On a generalization of Marcel Riesz' potentials and the semi-groups generated by them, Comm. Sém. Math. Univ. Lund Tome Supplémentaire 1952, 72-81.

5. H. Kober, $A$ modification of Hilbert transforms, the Weyl integral and functional equations, J. London Math. Soc. 42 (1967), 42-50.

6. G. O. Okikiolu, Fourier transforms and the operator $H_{\alpha}$, Proc. Cambridge Philos. Soc. 62 (1966), 73-78.

7. M. Riesz, L'integrale de Riemann-Liouville et le problème de Cauchy, Acta Math. 81 (1948), 1-223.

8. L. von Wolfersdorf, Über eine Beziehung zwischen Integralen nicht ganzer Ordnung, Math. Z. 90 (1965), 24-28.

9. H. Weyl, Bemerkungen zum Begriff des Differentialquotienten gebrochener Ordnung, Vierteljschr. Naturforsch. Ges. Zürich. 62 (1917), 296-302.

The Technological University of Aachen, Germany 